\title{
Special issue: Brazilian School on Statistical Mechanics, 2002 (Escola Brasileira de Mecânica Estatística, EBME)
}

The 2002 version of the Brazilian School on Statistical Mechanics (Escola Brasileira de Mecânica Estatística, EBME), which is hoped to happen every two years, took place from February 18 to March 1st, at the Instituto de Física de São Carlos, University of São Paulo, in the relatively small town of São Carlos, about $250 \mathrm{~km}$ from São Paulo. It was conceived as an answer to the need of a thematic school in statistical mechanics, at a national level, in order to promote short graduate courses on a variety of subjects and research topics that are relatively recent and not so well represented in the country. The school consisted of four short courses of approximately 10 hours, attended by about 70 students from several regions of Brazil, and whose lecture notes are finally being published in this special issue of the Braz. J. Phys.

In the first week of the school, there were two courses: "Introduction to Bosonization" by Eduardo Miranda, from the University of Campinas, and "Introduction to Quantum Monte Carlo Simulations for Fermionic Systems", by Raimundo R. dos Santos, from the Federal University of Rio de Janeiro. In the second week, there were two other courses: "An Introduction to Numerical Methods in Low-Dimensional Quantum Systems", by André L. Malvezzi, from the Universidade Estadual Paulista, in Bauru, São Paulo, and "Non-Equilibrium Phase Transitions", by Ronald Dickman, from the Federal University of Minas Gerais, in Belo Horizonte. The highly pedagogical notes of Eduardo Miranda, which are selfcontained and assume just some basic knowledge of second quantization, are of particular relevance as an introduction to the use of the modern bosonization techniques. Raimundo Santos presented a tutorial introduction to Monte Carlo techniques for simulations of classical and quantum systems, with particular emphasis on the determinantal quantum Monte Carlo method. This short course was designed to provide the necessary tools for carrying out a numerical analysis of fermionic systems such as the well-known Hubbard model. André Malvezzi presented a comprehensive and systematic overview, including details of implementation, of the Lanczos and the Density Matrix Renormalization methods, which are by far the most common numerical techniques to obtain the eigenspectra of quantum chains. In the last short course, Ronald Dickman presented some applications of several techniques (mean-field, series expansions, renormalization-group) to the analysis of non-equilibrium phase transitions in stochastic systems. Since most of these topics had already been covered in a recent book, Dickman decided to send us a paper on path integrals and perturbation theory for stochastic processes, with a number of applications for nonequilibrium systems.

Francisco C. Alcaraz

and Silvio R. A. Salinas

São Carlos, February, 2003. 